\title{
ICTIOFAUNA ASSOCIADA A MACRÓFITAS AQUÁTICAS DE AGUA LÊNTICA (LAGO CATALÃO) E LÓTICA (ILHA MARCHANTARIA) NA ÉPOCA DE VAZANTE, AMAZÔNIA CENTRAL
}

\author{
Cívil Prisyla CASADO DEL CASTILLO ${ }^{1,2}$, Michele MARQUES DE SOUZA², Daniele FERREIRA \\ CAMPOS $^{2}$, Karla Katriny DO AMARAL SERIQUE ${ }^{2}$, Jansen Alfredo SAMPAIO ZUANON ${ }^{2}$. \\ 1 Instituto de Investigaciones de la Amazonía Peruana - IIAP, Programa AQUAREC. Carretera Iquitos-Nauta Km 4,5. San \\ Juan Bautista, Loreto, Perú. E-mail pccbio@hotmail.com \\ 2 Biologia de Agua Doce e Pesca Interior - BADPI, Instituto Nacional de pesquisas da Amazônia - INPA. Av. André Araújo \\ 2936, Aleixo, Caixa postal 478, CEP 69060-001 Manaus, AM, Brasil.
}

\begin{abstract}
RESUMO
O objetivo do presente estudo foi avaliar a abundancia e riqueza da ictiofauna associada à macrófitas aquáticas de um corpo de agua lêntico (lago Catalão) e outro lótico (Ilha da Marchantaria), na época de vazante do rio Solimões. Os bancos de macrófitas apresentaram composição mista com uma área média de $13.36 \mathrm{~m}^{2}$. O total de peixes coletados em ambos os ambientes foi de 1.786 exemplares, distribuídos em 08 ordens, 23 famílias e 84 espécies. A ordem Characiformes foi a mais representativa tanto em riqueza de espécies quanto em abundância em ambos os ambientes. No lago Catalão foram coletados 1.087 exemplares distribuídos em 39 espécies, sendo as mais abundantes Mesonauta festivus (43.15\%), Hyphessobrycon melanochrous (23.18\%), e Serrasalmus maculatus (10\%). Para a Ilha da Marchantaria foram amostrados 699 indivíduos distribuídos em 67 espécies, das quais as mais abundantes foram Prionobrama filigera (24.03\%), Schizodon fasciatus (8.58\%) e Aphyocharax alburnos (7.15\%). O nível de similaridade foi maior entre os bancos das macrofitas de um mesmo ambiente (20\% a $70 \%$ ) que entre os ambientes $(10 \%)$. Segundo o teste t, não houve diferença significativa em relação à abundância entre os ambientes, porém, quanto à riqueza, $o$ ambiente lótico apresentou o maior número de espécies. Os resultados indicam que existe uma grande diversidade de peixes nos bancos de macrófitas no ambiente aquático lêntico e lótico, com maior numero de espécies em ambientes lóticos e maior numero de exemplares no ambiente lêntico.
\end{abstract}

PALAVRAS-CHAVE: Macrófitas, ictiofauna, ambiente lêntico, ambiente lótico.

\section{ICHTYOFAUNA ASSOCIATED WITH AQUATIC MACROPHYTES OF LENTIC (CALATAO LAKE) AND LOTIC (MARCHANTARIA ISLAND) WATER IN THE EPOCH OF EBB, CENTRAL AMAZON}

The objective of this study was to evaluate the abundance and richness of the fish fauna associated with macrophytes of a lentic body of water (Catalão Lake) and other lotic (Marchantaria Island), at the time of low tide, Solimões River. The banks of macrophytes showed mixed composition with an average area of $13.36 \mathrm{~m}^{2}$. The total number of fish collected in both environments was of 1.786 specimens, distributed in 8 orders, 23 families and 84 species. The order Characiformes was the most representative both in species richness and in abundance in both environments. The Catalão lake were collected 1.087 specimens distributed in 39 species, the most abundant were Mesonauta festivus (43.15\%), Hyphessobrycon melanochrous (23.18\%) and Serrasalmus maculatus (10\%). For the Marchantaria Island were sampled 699 individuals in 67 species, of which the most abundant were Prionobrama filigera (24.03\%), Schizodon fasciatus (8.58\%) and Aphyocharax alburnus (7.15\%). The level of similarity was highest among banks of macrophytes in the same environment (20 to $70 \%$ ) than among environments $(10 \%)$. According to t-test, no significant difference in abundance between the environments, however, as to richness, lotic had the largest number of species. The results indicate that there is a great diversity of fish in macrophyte aquatic of lotic and lentic environments, with the largest number of species in lotic and more specimens in lentic environments.

KEYWORDS: Macrophytes, fish fauna, environment lentic and lotic. 


\section{INTRODUÇÃO}

A presença de macrófitas aquáticas nos diferentes ecossistemas de água doce é um dos principais fatores ligados à estruturação dos habitats ocupados por animais aquáticos e terrestres (Junk, 1970, 1973; Esteves, 1986). Estes habitats são considerados os principais produtores de biomassa nos ecossistemas aquáticos, tendo um papel importante na dinâmica dos nutrientes, afetando a diversidade biológica, as relações interespecíficas e a produtividade do sistema (Agostinho et al., 2003). Assim também, as macrófitas abrigam, principalmente, peixes juvenis ou de pequeno porte e são consideradas, por vários autores, como áreas "berçário" ou criadouros por providenciarem abrigo contra predadores e alimento em abundância aos organismos que os habitam (Boehlert \& Mundy, 1988; Sogard, 1992; Felix et al., 2006; Falcão, 2007).

Os ambientes aquáticos amazônicos apresentam variações nos parâmetros físico-químicos, tais como na temperatura e oxigênio (Peters \& Nelson, 1987) profundidade e turbulência (Lasiak, 1986) e a correnteza (Ayvazian \& Hyndes, 1995) que podem influenciar na presença da ictiofauna associadas a estas macrófitas aquáticas (Queiroz et al., 2006), inclusive, este ultimo parâmetro define a característica de lêntico e lótico aos ambientes aquáticos amazônicos (Lowe-McConnell, 1999). O grau de correnteza é uns dos fatores que influenciam nos nível de oxigênio e turbidez de um corpo de água, pelo que são considerados os parâmetros responsáveis pelas mudanças na composição da ictiofauna e na dinâmica das interações entre os organismos nos dois tipos de ambientes, lêntico e lótico (J.Zuanon, com. pess.).

Em definição, os ambientes lênticos são locais de águas quase paradas ou com fluxo variável (lagos, lagoas, represas, reservatórios) e com entrada de energia principalmente de origem autóctone (Horne \& Goldman, 1994). Nestes ambientes se observam extensas regiões litorâneas, colonizadas por densas comunidades de macrófitas aquáticas, o que tornam a este compartimento um dos mais produtivos e com muitos habitats na maioria dos ecossistemas aquáticos continentais (Esteves, 1998). Enquanto que, os ambientes lóticos são locais de águas correntes como riachos e rios (Lowell-McConell, 1999) considerados doadores e receptores de nutrientes de origem alóctone, e à medida que o rio torna-se mais largo, estes nutrientes são substituídos por itens bênticos e planctônicos autóctones, sem os quais as cadeias alimentares não poderiam ser sustentadas (Vannote et al., 1980; Jeffries, 2000).

No ambiente lótico as herbáceas aquáticas, principalmente plantas enraizadas, promovem a redução da velocidade da água, tornando-os mais habitáveis por invertebrados, que podem servir de alimentos para os peixes (Templeton, 1995). Nos estudos sobre a diversidade biológica de peixes amazônicos torna-se importante avaliar as características que compõem os diferentes habitats que apresentam os ambientes aquáticos $\mathrm{e}$ as diferentes espécies que os habitam, para assim obter dados mais precisos sobre a composição biológica amazônica. Atualmente não existem dados sobre a diferença na composição da ictiofauna entre o lago Catalão e a Ilha Marchantaria, considerados como ambientes lênticos e lóticos respectivamente, pelo que, o presente estudo teve como objetivo avaliar a riqueza e a abundância da ictiofauna associada aos bancos de macrófitas aquáticas entre um ambiente lêntico (lago Catalão) e um ambiente lótico (Ilha da Marchantaria) do rio Solimões, Amazônia central, Brasil.

\section{MATERIAL E MÉTODOS CARACTERIZAÇÃO DA ÁREA DE ESTUDO}

Este estudo foi conduzido durante o período de vazante (agosto de 2010), no lago Catalão e nas proximidades da Ilha da Marchantaria do rio Solimões, a três e quinze $\mathrm{km}$ de distancia do Porto da Ceasa em Manaus, Estado do Amazonas-Brasil, respectivamente.

O lago Catalão é considerado ambiente lêntico e se situa próximo à confluência dos rios Solimões e Negro. Periodicamente é submetido a inundações de aporte de águas de ambos os rios, conectando-se ao rio Solimões durante o período de enchente e comumente conectado ao rio Negro ao longo do ano (Leite et al., 2006). Este ambiente apresenta alta concentração de nutrientes sedimentários e observam-se frequentemente densas áreas ocupadas por herbáceas aquáticas (Esteves, 1998). Além disso, Leite et al. (2006) mencionam que o lago Catalão é um importante berçário para as larvas dos peixes que desovam no rio Solimões e também para as larvas dos peixes que desovam no próprio lago, tornando-o um dos mais produtivos e com muitos habitats para a ictiofauna local.

Nas proximidades da Ilha da Marchantaria, rio Solimões, é caracterizada como ambiente lótico, a $10 \mathrm{~km}$ de distância do lago Catalão, aproximadamente. As margens desta ilha são ocupadas por grandes bancos de herbáceas aquáticas na época de vazante (Piedade et al., 1991). Em consequência do grande aporte de sedimentos erodidos da região andina, é um ambiente rico em nutrientes e apresenta condutividade elétrica elevada devido à alta concentração de íons dissolvidos (Sioli, 1984). 


\section{COLETADEDADOS}

Ao todo, foram definidos 15 bancos de macrófitas aquáticas em cada ambiente, com uma área média de $13.36 \mathrm{~m}^{2}$ e distância mínima aproximada de $100 \mathrm{~m}$ entre os bancos. Todas as coletas foram realizadas no período da manhã durante uma semana. Os bancos de macrófitas apresentaram composição mista com predominância das espécies Paspalum repens (membeca), Eichornia crassipes (mureru) e Salvinia auriculata (salvinia).

Para a captura dos peixes foi utilizada uma rede de cerco de $11 \mathrm{~m}$ de comprimento por $5 \mathrm{~m}$ de altura e malha de $5 \mathrm{~mm}$ entre nós opostos. Os peixes foram fixados em solução de formol a $10 \%$ e transportados em bandejas plásticas até o flutuante no lago Catalão do Instituto Nacional de Pesquisas da Amazônia. Os peixes foram posteriormente lavados e colocados em álcool a $70 \%$ para logo serem depositados na coleção de peixes do INPA. Para a identificação dos peixes foram utilizadas chaves taxonômicas e auxílio de um especialista.

\section{ANÁLISE ESTATÍSTICA}

Os dados foram apresentados em frequência relativa e absoluta do total de exemplares registrados e numero de espécies coletadas por ambiente estudado, lêntico e lótico. Para testar o nível de similaridade na composição da ictiofauna presente nos bancos de macrófitas aquáticas entre os ambientes, bem como o nível de similaridade entre os bancos de cada ambiente, foi utilizado o Índice de similaridade de Bray-Curtis. Para estimar a diferença da riqueza e abundância de espécies de peixes entre os ambientes foi utilizado o teste $t$ e a relação entre a área das herbáceas aquáticas com a riqueza da ictiofauna de cada ambiente foi utilizado análise de covariância (ANCOVA).

\section{RESULTADOS E DISCUSSÃO}

No presente estudo foi coletado um total de 1.786 exemplares de peixes de ambos os ambientes, lago Catalão e Ilha da Marchantaria. Os exemplares registrados foram classificados em oito ordens, 23 famílias e 84 espécies de peixes (Tabela 1 e 2), de pequeno e mediano porte, tanto espécies ornamentais como de consumo humano.

As ordens Characiformes e Perciformes foram as mais representativas em numero de exemplares do total coletado de ambos os ambientes (57.2 e 32.2\%, respetivamente). Estas ordens abrigam o maior numero de espécies que são de importância na pesca comercial e de subsistência na Amazônia Central (Santos et al., 2006), o que explicaria o maior numero de exemplares registrados para estas ordens no presente estudo. Além disso, o lago catalão e a
Ilha Marchantaria encontram-se próximos as áreas de desove de muitas espécies, principalmente de Characiformes (Araujo-Lima, 1984; Leite et al., 2006). Estes resultados coincide também com o observado por Sanchez-Botero et al. (2003); Pacheco (trabalho não publicado) e Prado et al. (2010), o que parece estar relacionada a condições ambientais como refúgio, oxigênio dissolvido e disponibilidade de alimento (Prado et al., 2010) assim como a capacidade visual dessas espécies em detectar predadores (Delariva et al., 1994) conduzindo os indivíduos a ambientes mais protegidos (Sánchez-Botero et al., 2003).

Embora as macrófitas aquáticas não sejam fontes de alimento importantes para os peixes a elas associadas (Forsberg et al., 1993) seus caules e raízes funcionam como substrato para o perifíton (Engle \& Melack, 1990) e abrigam ainda, uma grande quantidade de invertebrados (BlancoBelmont, 1990; Junk \& Robertson, 1990) que constituem importantes itens alimentares para juvenis de Characiformes migradores (Goulding \& Carvalho, 1982).

No lago catalão, considerado como ambiente lêntico, foram registradas 39 espécies com um total de 1087 exemplares, distribuídos em 12 famílias, das quais a familia Cichlidae (48.02\%) e Characidae $(42.87 \%)$ foram as que obtiveram maior representatividade. Neste ambiente, as espécies mais abundantes foram Mesonauta festivus, Hyphessobrycon melanochrous e Serrasalmus maculatus, representando $43.15 \% ; 23.18 \%$ e $10 \%$, respectivamente, do total de exemplares registrados (Tabela 2).

$\mathrm{Na}$ Ilha da Marchantaria (ambiente lótico), foram registradas 67 espécies com um total de 699 exemplares, distribuídos em 21 famílias, sendo as mais representativas Characidae $(46.35 \%)$ e Anastomidae (18.60\%). As espécies mais abundantes foram Prionobrama filigera, Schizodon fasciatus e Aphyocharax alburnus, representando $24.03 \% ; 8.58 \%$ e $7.15 \%$ respectivamente.

Vinte e duas espécies foram comuns aos dois ambientes representados principalmente por juvenis $(90 \%)$ e espécies de pequeno porte. As vinte e duas espécies comuns aos dois ambientes (Tabela 3) apresentam ampla distribuição geográfica e diversas adaptações biológicas (Sazima \& Zamprogno, 1985; Agostinho et al., 2007), além de serem comuns em biótipos de bancos de macrófitas aquáticas, permanecendo principalmente nas áreas periféricas, onde há maior oxigênio disponível (LowelMcConnell, 1999). Segundo o mesmo autor, em diversos estudos realizados na Amazônia, os valores de frequência relativa da maioria das espécies de peixes encontram-se abaixo de $1 \%$, como 
demonstrado neste estudo (Tabela 2). Segundo Santos \& Ferreira (1999) citado por LowelMcConnell (1999), em condições naturais de ecossistemas tropicais, as espécies estão distribuídas equitativamente, isto é, numerosas espécies são encontradas com pequeno número de exemplares cada.

O Índice similaridade de Bray-Curtis (Figura 1) indicou que, em geral, a similaridade na composição da ictiofauna foi apenas de $10 \%$ entre os ambientes lêntico e lótico, separando claramente a ictiofauna nos bancos das macrófitas aquáticas de cada ambiente. No entanto, a composição da ictiofauna entre as macrófitas aquáticas de um mesmo ambiente variou de 20 a $70 \%$ de similaridade, resultando em bancos homogêneos dentro de um mesmo ambiente.

Não houve diferença significativa na abundância de peixes entre os ambientes lêntico e lótico $\left(\mathrm{t}_{0.05(2)} 28=1,78 ; \mathrm{p}>0,05\right)$. Este fato pode estar relacionado ao período de vazante, época em que o presente trabalho foi desenvolvido. Neste período a disponibilidade de hábitats tende a reduzir, concentrando a biota aquática nos bancos de macrófitas das zonas litorâneas dos lagos e rios, especialmente para espécies que usam estes hábitats como refúgio (Arrigton et al., 2005). Por outro lado, a riqueza foi significativamente maior no ambiente lótico $\left(\mathrm{t}_{0,05(2)} 28=-2,56 ; \mathrm{p}<0,05\right)$.

$\mathrm{O}$ ambiente lótico apresentou o maior número de espécies, o que pode estar associado ao fato que, as macrófitas presentes nestes ambientes apresentam maior robustez em seus caules e raízes, formando uma estrutura mais complexa que as das macrófitas do lago Catalão. Estas estruturas oferecem maior disponibilidade de alimento e uma barreira física mais eficiente, apresentando por tanto maior riqueza de espécies que bancos com estruturas menos complexas (Petry et al., 2003; Agostinho et al., 2007; Sánchez-Botero et al., 2008). Araújo-Lima et al. (1986) sugerem que espécies mais susceptíveis a predação, nestes caso juvenis, estariam procurando os ambientes mais complexos nas raízes das macrófitas para fugirem de predadores.

A análise de covariância mostrou que não há uma relação significativa entre a riqueza da ictiofauna e a área dos bancos das macrófitas $(\mathrm{F}=0,28 ; \mathrm{p}>0,05)$, resultando em amostras homogêneas. Prado et al. (2010) mencionam que a estrutura e arquitetura dos estandes de macrófitas podem influenciar a distribuição da ictiofauna. No presente estudo, foram escolhidos bancos de macrófitas de composição e tamanho aparentemente homogêneo para evitar influência sobre a ictiofauna, o que foi corroborado pela analise de covariância indicando não haver relação entre essas variáveis.

Em resumo, o nível de correnteza de um ambiente aquático amazônico, rios e lagos, influenciam diretamente sobre a composição dos bancos de macrófitas nestes ambientes, que por sua vez, influenciam na riqueza e na abundancia de peixes, principalmente de larvas e juvenis para o refugio e alimentação, existindo maior numero de riqueza de peixes no ambiente lótico e maior abundancia o numero de exemplares no ambiente lêntico. Sugerindo que, em ambientes lóticos o deslocamento rápido dos bancos de macrófitas dificulta o estabelecimento das espécies, reduzindo o número de exemplares. Enquanto que no lago Catalão, por ser corpo de agua lêntico abriga maior numero de exemplares que se fixam no lugar para sua reprodução e alimentação, provavelmente especificas para algumas espécies. 
Tabela 1. Numero de espécies, por ordens, registrados no lago catalão (ambiente lêntico) e llha Marchantaria (ambiente lótico) na Amazônia Central.

\begin{tabular}{lcc}
\multicolumn{1}{c}{ ORDENS } & Lago Catalão & Ilha Marchantaria \\
\hline CHARACIFORMES & 25 & 30 \\
CLUPEIFORMES & 0 & 1 \\
CYPRINODONTIFORMES & 0 & 1 \\
GYMNOTIFORMES & 3 & 10 \\
\hline PERCIFORMES & 6 & 9 \\
\hline SILURIFORMES & 4 & 14 \\
\hline SYNBRANCHIFORMES & 1 & 1 \\
\hline TETRAODONTIFORMES & 0 & 1 \\
\hline $\mathrm{N}^{\circ}$ de ordens & 5 & 8 \\
\hline $\mathrm{N}^{\circ}$ de Famílias & 12 & 21 \\
\hline $\mathrm{N}^{\circ}$ de exemplares & 1.087 & 699 \\
\hline
\end{tabular}

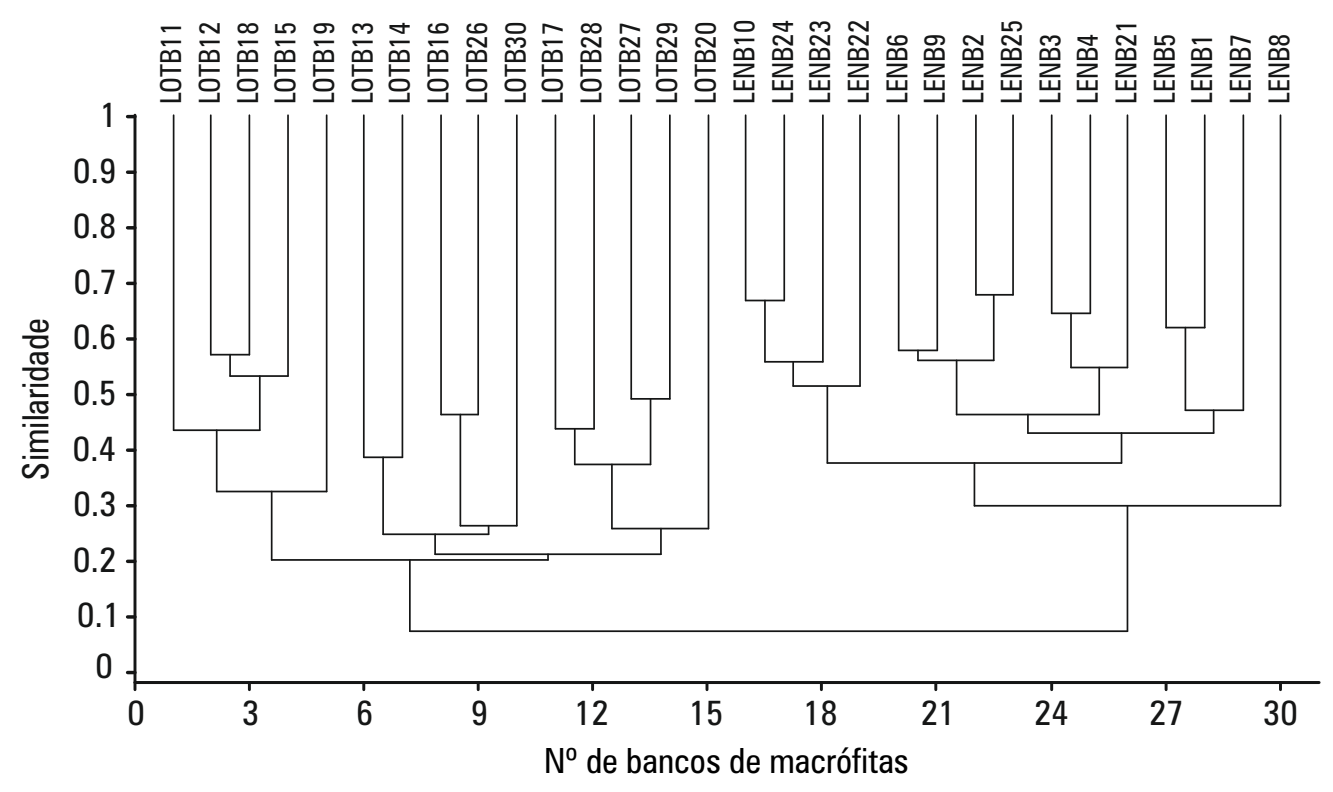

Figura 1. Dendrograma de similaridade (Bray-Curtis) entre os bancos de macrófitas dos ambientes lêntico (Catalão) e lótico (llha Marchantaria), Amazonas. (Len = Lêntico; Lot = Lótico; $\mathrm{B}_{\mathrm{n}}=$ número de bancos) 
Tabela 2. Lista de espécies e frequência relativa (\%) da ictiofauna associada a bancos de macrófitas dos ambientes lêntico (lago Catalão - LC) e lótico (Ilha da Marchantaria - IM).

\begin{tabular}{|c|c|c|c|c|}
\hline ORDEM & FAMILIA & ESPECIES & $\begin{array}{r}\text { LC } \\
\% \\
\end{array}$ & $\begin{array}{c}\text { IM } \\
\% \\
\end{array}$ \\
\hline \multirow{41}{*}{ CHARACIFORMES } & \multirow{6}{*}{ Anostomidae } & Abramites hypselonotus (Günther, 1868) & - & 4.72 \\
\hline & & Laemolyta proxima (Garman, 1890) & 0.55 & 0.14 \\
\hline & & Leporinus fasciatus (Bloch, 1794) & - & 0.86 \\
\hline & & Leporinus friderici (Bloch, 1794) & - & 2.29 \\
\hline & & Rhythiodus microlepis (Kner, 1858) & 0.74 & 2 \\
\hline & & Schizodon fasciatus (Agassiz e Spix, 1829) & 0.55 & 8.58 \\
\hline & Acestrorhynchidae & Acestrorhynchus falcirostris (Cuvier, 1819) & 0.09 & - \\
\hline & \multirow{27}{*}{ Characidae } & Aphyocharax alburnus (Günther, 1869) & - & 7.15 \\
\hline & & Aphyocharax avary (Fowler, 1913) & 0.09 & 2.29 \\
\hline & & Astyanax bimaculatus (Linnaeus, 1758) & - & 0.29 \\
\hline & & Charax sp. & 0.09 & 0.14 \\
\hline & & Ctenobrycon hauxwellianus (Cope, 1870) & 1.66 & 1.43 \\
\hline & & Hyphessobrycon melanochrous & 23.18 & - \\
\hline & & Hyphessobrycon eques (Steindachner, 1882) & 0.18 & 0.14 \\
\hline & & Metynis lippincottianus (Cope, 1870) & 0.28 & - \\
\hline & & Metynnis argenteus (Ahl, 1923) & 0.37 & - \\
\hline & & Moenkausia cf. lepidura (Kner, 1858) & - & 0.72 \\
\hline & & Moenkhausia collettii (Steindachner, 1882) & - & 0.29 \\
\hline & & Moenkhausia dichroura (Kner, 1858) & 0.46 & - \\
\hline & & Moenkhausia gracilima (Eigenmann, 1908) & 0.28 & - \\
\hline & & Moenkhausia robertsi (Géry, 1964) & - & 0.14 \\
\hline & & Myleus setiger (Müller \& Troschel, 1844) & - & 1.29 \\
\hline & & Mylossoma aureum (Agassiz, 1829) & - & 0.43 \\
\hline & & Mylossoma duriventre (Cuvier, 1818) & 1.1 & 3.86 \\
\hline & & Odontostilbe fugitiva (Cope, 1870) & 0.28 & 0.72 \\
\hline & & Paragoniates alburnus (Steindachner, 1876) & - & 0.14 \\
\hline & & Prionobrama filigera (Cope, 1870) & 0.46 & 24.03 \\
\hline & & Roeboides myersii (Gill, 1870) & - & 0.14 \\
\hline & & Serrapinus sp. & 1.84 & - \\
\hline & & Serrasalmus elongatus (Kner, 1858 ) & 0.18 & - \\
\hline & & Serrasalmus maculatus (Kner, 1858) & 10.03 & 0.57 \\
\hline & & Serrasalmus rhombeus (Linnaeus, 1766) & 0.09 & 2.43 \\
\hline & & Serrasalmus sp. N & 0.09 & - \\
\hline & & Triportheus albus (Cope, 1872) & 2.21 & 0.14 \\
\hline & Curimatidae & Curimatella alburna (Müller \& Troschel, 1844) & - & 0.14 \\
\hline & Hemiodontidae & Hemiodus sp. & 0.09 & - \\
\hline & Erythrinidae & Hoplias malabaricus (Bloch, 1794) & 2.85 & 0.72 \\
\hline & Lebiasinidae & Pyrrhulina brevis (Steindachner, 1876) & 2.39 & 0.14 \\
\hline & Cynodontidae & Rhaphiodon vulpinus (Agassiz, 1829) & - & 0.29 \\
\hline & Gasteropelecidae & Thoracocharax stellatus (Kner, 1858) & - & 2 \\
\hline & Engraulididae & Lycengraulis batesii (Günther, 1868) & - & 0.14 \\
\hline
\end{tabular}


Continuação tabela 2...

\begin{tabular}{|c|c|c|c|c|}
\hline ORDEM & FAMILIA & ESPECIES & $\begin{array}{l}\text { LC } \\
\% \\
\end{array}$ & $\begin{array}{c}\text { IM } \\
\%\end{array}$ \\
\hline CYPRINODONTIFORMES & Rivulidae & Rivulus obscurus (Garman, 1895) & - & 0.14 \\
\hline \multirow{11}{*}{ GYMNOTIFORMES } & \multirow{5}{*}{ Apteronotidae } & Adontosternachus clarkae (Lundberg \& Baskin, 1985) & - & 0.57 \\
\hline & & Apteronotus albifrons (Linnaeus, 1766) & - & 0.14 \\
\hline & & Apteronotus bonapartii (Castelnau, 1855) & - & 3.72 \\
\hline & & Parapteronotus hasemani (Ellis, 1913) & - & 1 \\
\hline & & Sternarchogiton nattereri (Steindachner, 1868) & - & 0.72 \\
\hline & \multirow{4}{*}{ Hypopomidae } & Brachyhypopomus brevirostris (Steindachner, 1868) & - & 0.43 \\
\hline & & Brachyhypopomus pinnicaudatus (Hopkins, 1991) & 0.09 & 0.14 \\
\hline & & Brachyhypopomus sp. & 0.09 & - \\
\hline & & Steatogenys elegans (Steindachner, 1880) & - & 0.29 \\
\hline & \multirow{2}{*}{ Sternopygidae } & Eigenmamnia aff. Trilineata (López \& Castello, 1966) & 0.46 & 0.29 \\
\hline & & Eigenmannia macrops (Boulenger, 1897) & - & 1.14 \\
\hline \multirow{10}{*}{ PERCIFORMES } & \multirow{10}{*}{ Cichlidae } & Apistogramma agassizii (Steindachner, 1875) & - & 0.43 \\
\hline & & Cichla monoculus (Spix e Agassiz, 1831) & 1.2 & 0.14 \\
\hline & & Cichlasoma amazonarum (Kullander, 1983) & - & 1.43 \\
\hline & & Crenicichla inpa (Ploeg, 1991) & - & 0.29 \\
\hline & & Crenicichla reticulata (Heckel, 1840) & - & 0.14 \\
\hline & & Crenicihla cincta (Regan, 1905) & 0.18 & 0.72 \\
\hline & & Heros efasciatus (Heckel, 1840) & 0.46 & 0.29 \\
\hline & & Heros spurius (Heckel, 1840) & 0.46 & 0.29 \\
\hline & & Mesonauta festivus (Heckel, 1840) & 43.15 & 3.86 \\
\hline & & Pterophyllum scalare (Schultze, 1823) & 2.58 & - \\
\hline \multirow{18}{*}{ SILURIFORMES } & \multirow{3}{*}{ Auchenipiteridae } & Centromochlus heckelii (De Filippi, 1853) & - & 0.29 \\
\hline & & Parauchenipterus galeatus (Linnaeus, 1766) & - & 0.14 \\
\hline & & Parauchenipterus porosus (Bleeker, 1862) & 0.46 & - \\
\hline & \multirow{4}{*}{ Doradidae } & Anadoras grypus (Cope, 1872) & 0.18 & - \\
\hline & & Nemadoras humeralis (Kner, 1855) & 0.28 & - \\
\hline & & Oxydoras eigenmanni (Boulenger, 1895) & 0.09 & - \\
\hline & & Pterodoras granulosus (Valenciennes, 1821) & - & 1 \\
\hline & Heptapteridae & Pimelodella aff. cristata (Müller \& Troschel, 1849) & - & 3.15 \\
\hline & \multirow{7}{*}{ Loricariidae } & Farlowella oxyrhyncha & - & 1.43 \\
\hline & & Hypoptopoma gulare (Cope, 1878) & - & 4.01 \\
\hline & & Hypostomus aff. plecostomus (Linnaeus, 1758) & - & 0.72 \\
\hline & & Peckoltia brevis (La Monte, 1935) & - & 0.14 \\
\hline & & Rineloricaria castroi (Isbrücker \& Nijssen, 1984) & - & 0.29 \\
\hline & & Squaliforma emarginata (Nalenciennes, 1840) & - & 0.86 \\
\hline & & Sturisoma sp. & - & 0.43 \\
\hline & \multirow{2}{*}{ Pimelodidae } & Pimelodus cf. blochii (Valenciennes, 1840) & - & 0.43 \\
\hline & & Sorubim lima (Bloch \& Schneider, 1801) & - & 0.14 \\
\hline & Pseudopimelodidae & Microglanis cf. poecilus (Eigenmann, 1912) & - & 0.14 \\
\hline \multirow{2}{*}{ SYNBRANCHIFORMES } & \multirow{2}{*}{ Synbranchidae } & Synbranchus madeirae (Rosen \& Rumney, 1972) & - & 0.29 \\
\hline & & Synbranchus sp. & 0.18 & - \\
\hline TETRAODOTIFORMES & Tetraodontidae & Colomesus asellus (Müller eTroschel, 1848) & - & 2 \\
\hline
\end{tabular}


Tabela 3: Numero de espécies comuns entre ambos os ambientes lêntico (lago Catalão - LC) e lótico (llha da Marchantaria - IM).

\begin{tabular}{|c|c|c|c|}
\hline FAMILIA & ESPECIES & LC & IM \\
\hline \multirow{3}{*}{ Anostomidae } & Laemolyta proxima (Garman, 1890) & 0.55 & 0.14 \\
\hline & Rhythiodus microlepis (Kner, 1858) & 0.74 & 2 \\
\hline & Schizodon fasciatus (Agassiz e Spix, 1829) & 0.55 & 8.58 \\
\hline \multirow{10}{*}{ Characidae } & Aphyocharax avary (Fowler, 1913) & 0.09 & 2.29 \\
\hline & Charax sp. & 0.09 & 0.14 \\
\hline & Ctenobrycon hauxwellianus (Cope, 1870) & 1.66 & 1.43 \\
\hline & Hyphessobrycon eques (Steindachner, 1882) & 0.18 & 0.14 \\
\hline & Mylossoma duriventre (Cuvier, 1818) & 1.1 & 3.86 \\
\hline & Odontostilbe fugitiva (Cope, 1870) & 0.28 & 0.72 \\
\hline & Prionobrama filigera (Cope, 1870) & 0.46 & 24.03 \\
\hline & Serrasalmus maculatus (Kner, 1858) & 10.03 & 0.57 \\
\hline & Serrasalmus rhombeus (Linnaeus, 1766) & 0.09 & 2.43 \\
\hline & Triportheus albus (Cope, 1872) & 2.21 & 0.14 \\
\hline Erythrinidae & Hoplias malabaricus (Bloch, 1794) & 2.85 & 0.72 \\
\hline Lebiasinidae & Pyrrhulina brevis (Steindachner, 1876) & 2.39 & 0.14 \\
\hline Hypopomidae & Brachyhypopomus pinnicaudatus (Hopkins, 1991) & 0.09 & 0.14 \\
\hline Sternopygidae & Eigenmamnia aff. Trilineata (López \& Castello, 1966) & 0.46 & 0.29 \\
\hline \multirow{5}{*}{ Cichlidae } & Cichla monoculus (Spix e Agassiz, 1831) & 1.2 & 0.14 \\
\hline & Crenicihla cincta (Regan, 1905) & 0.18 & 0.72 \\
\hline & Heros efasciatus (Heckel, 1840) & 0.46 & 0.29 \\
\hline & Heros spurius (Heckel, 1840) & 0.46 & 0.29 \\
\hline & Mesonauta festivus (Heckel, 1840) & 43.15 & 3.86 \\
\hline
\end{tabular}




\section{BIBLIOGRAFIA CITADA}

Agostinho, A.A.; Gomes, L.C.; Julio Jr, H.F. 2003. Relações entre macrófitas e fauna de peixes. In: Thomaz, S. M., Bini, L.M. (Eds.), Ecologia e Manejo de Macrófitas Aquáticas. 261-279.

Agostinho, A. A.; Thomaz, S. M.; Gomes, L. C.; Baltar, S.L.S.M.A. 2007. Influence of the macrophyte Eichhornia azurea on fish assemblage of the upper Paraná River floodplain (Brazil). Aquatic Ecology, 41: 611619.

Araújo-Lima, C.A.R.M. 1984. Distribuição espacial e temporal de larvas de Characiformes em um setor do rio Solimões/Amazonas próximo a Manaus, AM. Dissertação de Mestrado, Instituto Nacional de Pesquisas da Amazônia/Fundação Universidade do Amazonas, Manaus, estado do Amazonas, Brasil. 84pp.

Araújo-Lima, C. A.; Portugal, L. P. S.; Ferreira, E. G. 1986. Fish-macrophytes relationship in the Anavilhanas Archipelago, a black-water system in the central Amazon. Journal of Fish Biology, 29: 1-11.

Arrington, D. A.; Winemiller, K. O.; Layman, C.A. 2005. Community assembly at the patch scale in a specie rich tropical river. Oecologia, 144: 157167.

Ayvazian S.G.; Hyndes G.A. 1995. Surf-zone fish assemblages in South-westem Australia: do adjacent nearshore habitats and the warm Leeuwin Current influence the characteristics of the fish fauna?. Marine Biology. 122: 527-536.

Blanco-Belmont, L. 1990. Estudio de las comunidades de invertebrados asociados a las macrófitas acuáticas de tres lagunas de inundación de la sección baja del Orinoco. Sociedad de Ciencias Naturales La Salle, 71107.

Boehlert, G.W.; Mundy, B.C. 1988. Roles of behavioral and physical factors in larval and juvenile fish recruitment to estuarine nursery areas. In: Weinstein, M.P, editor. Larval fish and shellfish transport through inlets. American fisheries society Symposium. 3: 51-67.

Delariva, R.L.; Agostinho, A.A.; Nakatani, K.; Baumgartner, G. 1994. Icthyofauna associated aquatic macrophytes in the upper Parana River floodplain. Revista UNIMAR, 16(3): 41-60.

Engle, D.L.; Melack, J.M. 1990. Floating meadow epiphyton: Biological and chemical features of epiphytic material in an Amazonian floodplain lake. Freshwater Biology, 23: 479-494.

Esteves, F.A.; Camargo, A.F.M. 1986. Sobre o papel das macrófitas aquáticas na estocagem e ciclagem de nutrientes. Acta de limnologia, 6: 273-298.
Esteves, F.A. 1998. Fundamentos de Limnologia. 2ed. Interciência: Rio de Janeiro.

Forsberg, B.R; Araújo-Lima, C.A.R.M.; Martinelli, L.A.; Victoria, R.L.; Bonassi, J.A. 1993. Autotrophic carbon sources for fish of the Central Amazon. Ecology, 74: 507-515.

Golding, M.; Carvalho, M. 1982. Life history and management of tambaqui (Colossoma macropomum, Characidae): An important Amazonian food fish. Revista Brasileira de Zoologia, 1: 107-133.

Jeffries, R.L. 2000. Allocthonous inputs: integrating populations changes and food-web dynamics. Tree, 15(1):19-22.

Junk, W.J. 1970. Investigations on the ecology and production biology of the 'floating meadows' (Paspalo echinochloetum) on the middle Amazon part I: the floating vegetation and its ecology. Amazoniana II, 449 - 495.

Junk, W.J. 1973. Investigations on the ecology and production biology of the 'floating meadows' (Paspalo echinochloetum) on the middle Amazon part II: the Aquatic fauna in the root zone of floating vegetation. Amazoniana IV, 9102.

Junk, W.J.; Robertson, B. 1990. Aquatic invertebrates. In: Junk, W.J. (Ed.) The Central Amazonian floodplain: Ecology of a pulsing systems. Ecological Studies. Springer Verlag, 26: 279-298.

Lasiak, T.A. 1986. The reproductive cycles of the intertidal bivalves Crassostrea cucullata (Born, 1778) and Perna perna (Linnaeus, 1758) from the Transkei coast, southern Africa. Veliger, 29: 226-230

Leite, R.G.; Silva, J.V.V.; C.E. Freitas. 2006. Abundância e distribuição das larvas de peixes no Lago Catalão e no encontro dos rios Solimões e Negro, Amazonas, Brasil, Acta amazônica. 36(4): 557-562.

Lowe-McConnell, R.H. 1999. Estudos ecológicos de comunidades de peixes tropicais. Vazzoler, A.E.A.M.; Agostinho, A.A.; Cunnhingham, P.T.M. (tradud.)-EDUSP: São Paulo.

Peters, D.J.; Nelson, W.G.. 1987. The seasonality and spatial patterns of juvenile surf zone fishes of the Florida East Coast. Florida Scient, 50: $85-99$.

Petry, P; Bayley, P.B.; Markle, D.F. 2003. Relationships between fish assemblages, macrophytes and environmental gradients in the Amazon river floodplain. Journal of Fish Biology, 63 (3): 547-579.

Piedade, MT.F.; Junk, W.J.; Long, S.P. 1991. The production of the $\mathrm{C} 4$ grass Echinochloa Polystachya on the Amazon flooplain. Ecology, 72(4): 1456-1463. 
Prado, K.L.L.; Freitas, C.E.; Soares, M.G.M. 2010. Assembleias de peixes associadas a macrófitas em lagos de várzea do baixo rio Solimões. Biotemas, 23(1):131-142.

Queiroz, L.P.; Conceição, A.A.; Giulietti, A.M. 2006. Nordeste semi-árido: caracterização geral e lista das fanerógamas. In: Giulietti, A.M.; Conceição, A.A.; Queiroz, L.P. (Eds.). Diversidade e caracterização das fanerógamas do semi-árido brasileiro. Recife: Associação Plantas do Nordeste, 1: 15-40.

Sánchez-Botero, J.I.; Farias M.L.; Piedade, M.T.; Garcez, D.S. 2003. Ictiofauna associada às macrofitas aquáticas Eichhornia azurea (SW.) Kunth. e Eichhornia crassipes (Mart.) Solms. No lago Camaleão, Amazônia Central, Brasil. Acta scientiarum, Biological Sciences, 25(2):369-375.

Sánchez-Botero, J. I.; Araújo-Lima, C.A.R.M.; Garcez, D.S. 2008. Effects of types of aquatic macrophyte stands and variations of dissolved oxygen and of temperature on the distribution of fishes in lakes of the Amazonian floodplain.
Acta Limnológica Brasileira, 20 (1): 45-54.

Santos, G; Ferrreira, E.; Zuanon, J. 2006. Peixes comerciais de Manaus. IBAMA/AM, ProVárzea, $144 \mathrm{p}$.

Sazima, I.; Zamprogno, C. 1985. Use of water hyacinths as shelter, foraging place, and transport by young piranhas Serrasalmus spilopleura. Environmental Biology of Fishes, 12:237-240.

Sioli, H. 1984. The Amazon: Limnology and landscape ecology of a mighty tropical river and its basin. Vol 56.763pp.

Sogard S.M. 1992. Variability in growth rates of juvenile fishes in different estuarine habitats. Mar Ecolgy, Prog Ser, 85:35-53.

Templenton, R. 1995. Freshwater fisheries management. Fishing News Books, Oxford, $241 \mathrm{pp}$.

Vannote, R.L., Minshall, G.W., Cummins, K.W., Sedell, J.R., Cushing, C.E. 1980. The river continuum concept. Can. J. Fish. Aquatic Sciences. 37(1):130-137. 